\title{
LA PORTADA DE LA IGLESIA DE SAN FERNANDO EN LA CIUDAD DE MEXICO. IMAGEN E IDEA
}

\section{RAFAel Cómez}

La imagen expresa los principios implícitos por los que una sociedad vive mientras que, por otra parte, comunica la comprensión de supuestos que a nivel popular no requieren demostración. La portada de la iglesia de San Fernando en la ciudad de México se nos ofrece como un singular ejemplo del imperio de la imagen en el arte virreinal de la Nueva España. El objeto de este estudio será, en primer lugar, presentar el grabado que sirvió de fuente de inspiración para el relieve de la portada de dicha iglesia y, en segundo lugar, analizar el papel ideológico de la imagen tras la metamorfosis artística del modelo.

La iglesia de San Fernando del colegio de Propaganda Fide de los franciscanos en México fue trazada por el famoso arquitecto Jerónimo Balbás, el introductor del estípite en México, con anterioridad a 1738. ${ }^{1}$ Su fachada es muy movida y la planta en saliente adopta una forma "abiombada" en la que se refleja la influencia de la basílica de Guadalupe, al mismo tiempo que el alzado muestra una estructura de dos cuerpos que remata en un tímpano con óculo octogonal. Una típica "fachada retablo" mexicana en cuyas calles laterales del primer cuerpo aparecen Santo Domingo y San Francisco entre columnas de estrías onduladas y en el segundo cuerpo, San José y San Antonio de Padua entre estípites con las efigies de los apóstoles San Bartolomé y Santiago, culminando en dos tondos con San Joaquín y Santa Ana que flanquean el óculo octogonal. Este despliegue iconográfico sirve de marco al tema central de la composición: el relieve que nos muestra al patrono de la iglesia, San Fernando, entre estípites con las efigies de San Pedro y San Pablo.

San Fernando se nos presenta con corona, manto, globo terráqueo y espada, según la iconografía típica hispalense, usada desde Pacheco a Roldán y Murillo. Sobre una colina que asoma a un mar de llamas donde se cuecen tres moros encadenados, calvos, barbudos y de nariz aguileña mientras otro con turbante se postra y hace una ofrenda, junto a otro barbudo con cetro en un plano inferior. En el fondo, acompañando al

1 D. Angulo, Historia del Arte Hispanomericano, II, Barcelona, 1950, p. 558; P. Rojas, Historia General del Arte Mexicano. Época Colonial, II, México, 2a. ed., 1981, p. 292; G. Tovar de Teresa, México barroco, México, 1981, p. 87. 
Santo Rey se ha representado a la Fortaleza abrazando una columna, en el lado izquierdo, y la Fe con la cruz y el cáliz, en el lado derecho. Sobre la figura de la primera aparece la Fama haciendo sonar la trompeta y dos ángeles con corona y palma sobrevuelan a Fernando III, flanqueados todos ellos por columnas corintias coronadas por globos o esferas.

Pedro Rojas interpretó el tema iconográfico de este relieve como San Fernando, abogado de las ánimas del purgatorio. ${ }^{2}$ Obviamente, no conocemos ninguna representación del Santo Rey en el purgatorio ni menos como abogado de almas de musulmanes que purgen sus pecados entre las llamas. Un examen detenido de los fondos de la librería del colegio franciscano de San Fernando de México - hoy ubicados en la Biblioteca Nacional de México- nos ha permitido identificar el grabado que sirvió de inspiración para el relieve, lo cual nos lleva a la intepretación que de ese modelo hizo el artista, introduciéndonos asimismo en el frondoso bosque de la bibliografía fernandina. ${ }^{3}$

2 P. Rojas, op. cit, p. 292.

3 B. García de Escañuela, Trono de glorias, adornado de sabios. panegyrico laudatorio de las heroycas virtudes, y victorias del rey D. Fernando el Santo, tercero de Castilla, y Leon. en la nueva, y primera ampliación de su culto, celebrada en la Real Capilla a siete de iunio de mil seiscientos y setenta y uno, Madrid, 1671; Iglesia Catolica. Congregatio Sacrorum Rituum. Eminentissimis, et reverendissimis DD. cardinalibus S. Congregationis Rituum. Proponente eminentissimo, et reverendissimo $D$. Card. Antonio Barberino, hispalen canonizationis servi Dei, gloriosissimi, inuictissimi, ac santi regis D. Ferdinandi III.. . Romae, 1638; Juan de la Presentación, El cavallero de Christo; vida de San Fernando, rey de España, (s.p.i.), (1678; Juan de San Agustín. Triumpho panegyrico. Aplauso real; celebra-

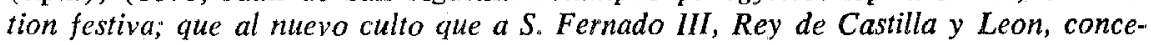
dio nuestro muy Santo Padre Clemente Decimo. Consagró la muy ilustre, augusta, santa, celebre, metropolitana, y patriarcal Iglesia de Sevilla, Sevilla, 1671; M. A. Laureti, Historia del glorioso D. Ferdinando Terzo, il Santo Rè delle Spagna, Napoli, 1680; A. Núñez de Castro, Vida de San Fernando el tercero rey de Castilla y Leon; ley viva de principes perfectos; desempeño de los precepios más seberos con que estrecharon a sus principes subditos, politicos y estadistas, Madrid, 1673, D. V. Papenbroeck, Acta vitae s. Ferdinandi, regis Castellae et Legionis ejus nominis tertil, cum postuma illius gloria, et hisioria s. crucis caravacanae, eoden quo ipse natus est anno $M$ CXCVIII caelitus allatae ex latinis et hispanicis coaevorum scriptis collecta, varieque illustrata, comentariis, annotationibus et iconibus, Antuerpiae, 1684; N. Sánchez, Sangre real virtuosa, coronada por N.SS.P. Clemente $X$, con el breue de la extensión de culto, y beatificacion de San Fernando rey de Castilla, y de Leon tercero de este nombre. Celebrada por el Real convento de $S$. Clara de la ciudad de Guadalaxara, Madrid, 1671; A de Solís y Rivadeneyra, Gloria póstuma en Sevilla de $S$ Fernando rey de España, desde su feliz tránsito, hasta la lultima translación de su incorrupto cuerpo el año 1729, Sevilla (s.a.). Todos estos libros ubicados en la Biblioteca Nacional de México pertenecieron a la librería del colegio franciscano de San Fernando de aquella ciudad. 


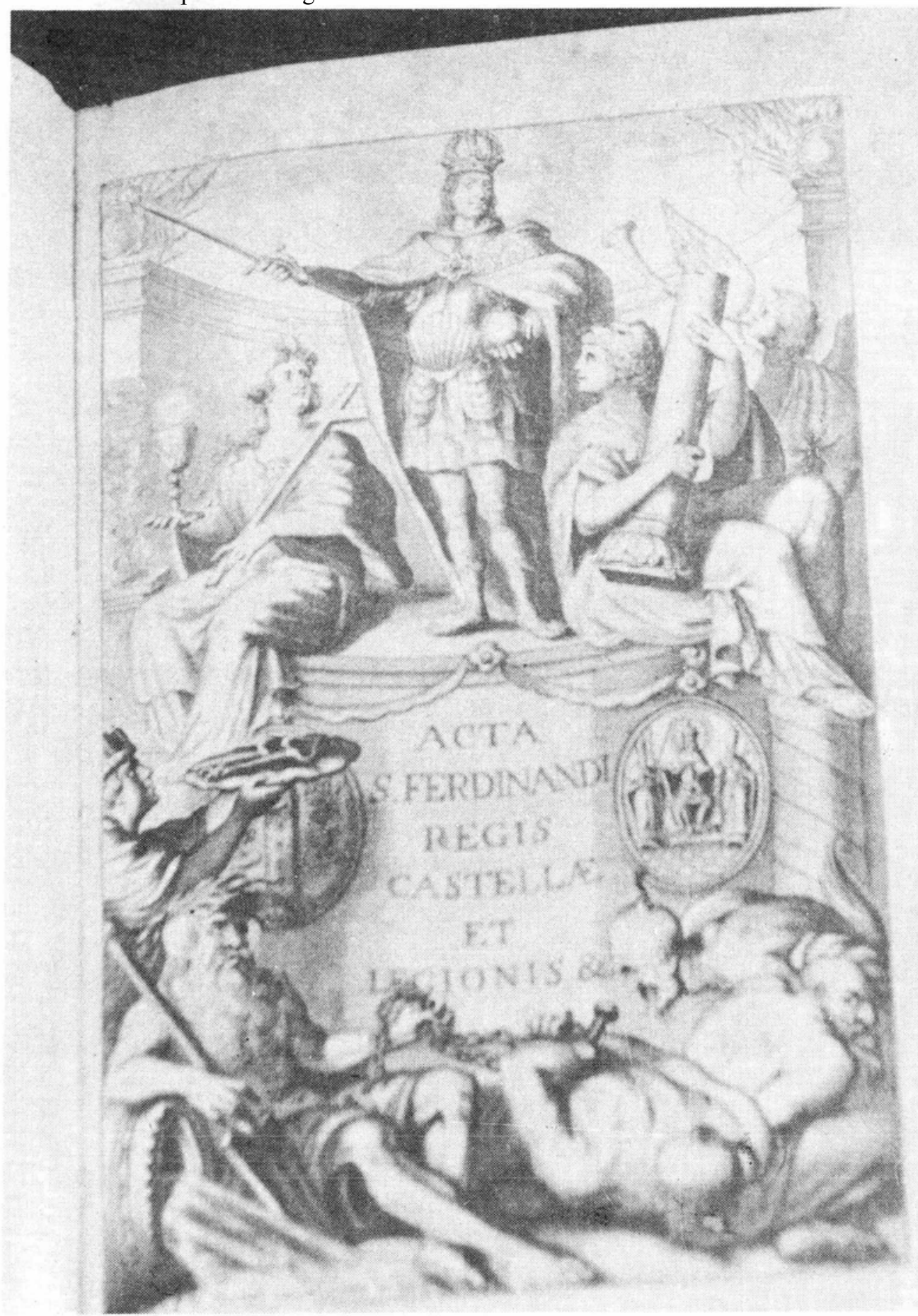

1. Philippe Bouttats el joven, grabado para el Acta Vitae S. Fernandi. Amberes, 1684 . 
DOI: http://dx.doi.org/10.22201/iie.18703062e.1989.60.1544

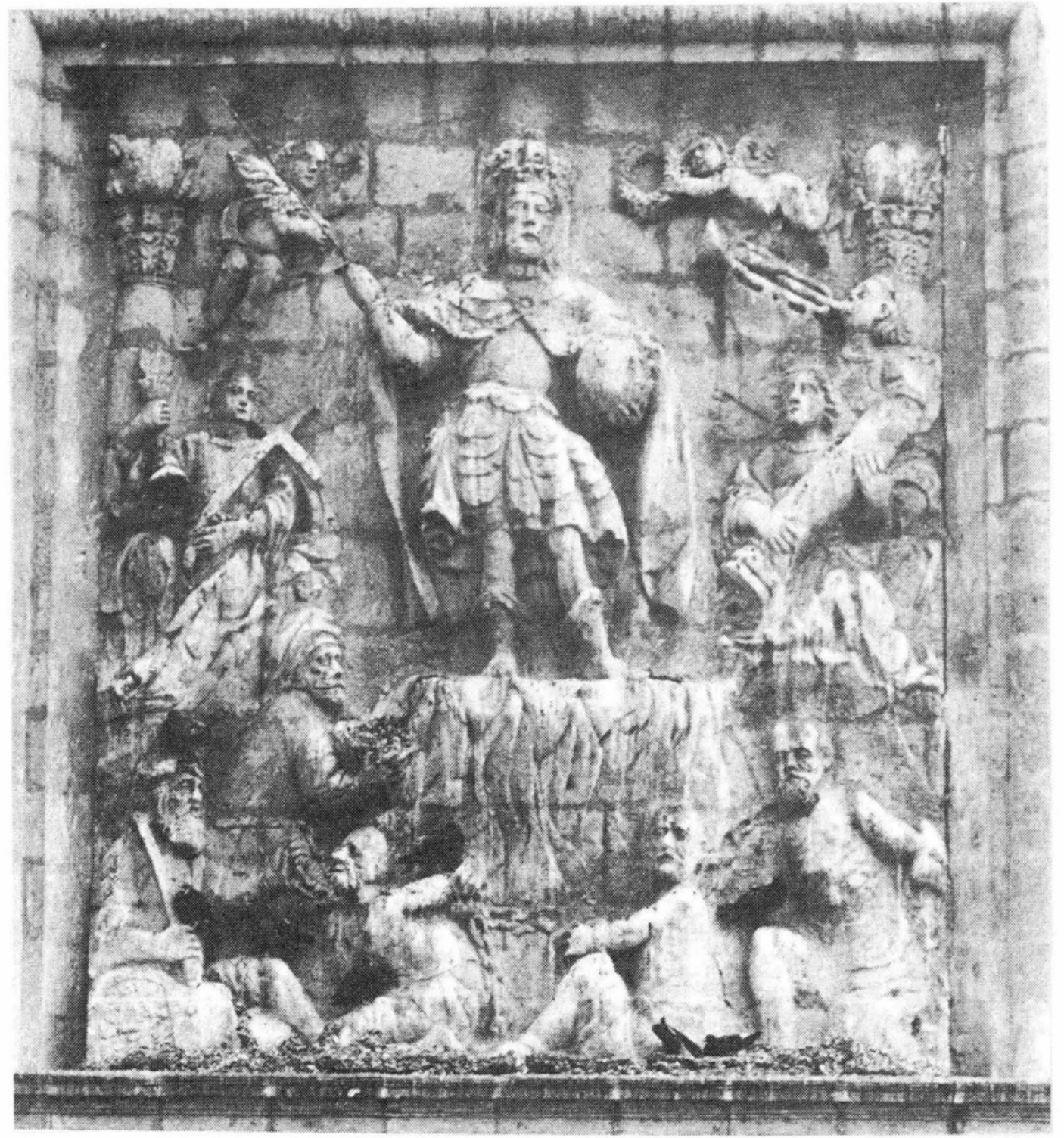

2. San Fernando. Relieve en la portada de la iglesia de San Fernando. México, D. F. Siglo XVIII. 
El grabado inicial que Philippe Bouttats el Joven realizó para la obra del jesuita Daniel Van Papenbroeck, Acta vitae S. Ferdinandi, publicada en Amberes en 1684, fue el modelo a seguir por el artista escultor. Allí aparece en primer término la representación del río Guadalquivir que encadena a los infieles -clara alusión a la conquista de Sevilla que, después de largo asedio, se decidió, finalmente, a través del río-. Tras la figura del río, el cadí Axataf entrega las llaves de la ciudad al conquistador. Éste, en un plano superior, sobre un podio del que penden los escudos de la Catedral y de la ciudad de Sevilla, aparece con coraza y manto real, espada y globo, mientras que un nimbo resplandeciente emana de su cabeza coronada. A ambos lados, sentadas sobre el podio, se encuentran, a la derecha, la Fe portando la cruz y el cáliz; a la izquierda, la Fortaleza, cubierta por piel de león y abrazada a una columna. La Fama soplando una trompeta queda en tercer plano, que cierra una exedra cuya cornisa muestra en sus extremos sendos trofeos militares, entre los que se destaca el escudo de la media luna.

Como puede observarse en el relieve de la portada de la iglesia de San Fernando, el artista encadenó entre sí a los tres moros del primer plano, dejó en el mismo ángulo al venerable Guadalquivir y transformó en turbante la corona del cadí Axataf. El podio se tornó en monte al que ascienden las llamas al tiempo que las mencionadas virtudes se separan algo del rey santo. Se destaca más la figura de la Fama y aparecen nuevos elementos: dos angelitos que revolotean con corona y palma en las manos, así como un par de columnas con capiteles corintios coronados por globos o esferas. Estos remates parecen haber sido imaginados en función de los trofeos que culminan los extremos de la cornisa en la exedra del modelo.

Curiosamente, este grabado de la obra de Papenbroeck era el que Interián de Ayala no recomendaba en su tratado de iconografía como modelo a imitar por no considerar esta representación "verdadera efigie", pues no le agradaba especialmente "porque las piernas y muslos están pintados al modo de los Romanos, y a lo militar, lo mismo que si se representara a la vista la imagen de César, o la de Pompeyo."4

Sin embargo, este aspecto heroico y militar como "miles christianus" fue el que se escogió para la portada de la iglesia del colegio de Propaganda Fide de los franciscanos en la ciudad de México. El aspecto he-

4 J. Interián de Ayala, Pictor christianus eruditus sive de Erroribus qui passim admittuntur circa pingendas, atque effigendas sacras Imagenes, Matritum, 1730 (2a. ed., 1782), II pp. 221-222. 
roico del personaje arranca de una idealizada visión de la conquista de Sevilla que tiene su origen en la poesía épica del siglo XVI. Aparte los fines estéticos y literarios, el tema épico de la conquista de Sevilla tenía como fin principal la apología y difusión de las virtudes de Fernando III y el elogio y genealogía de la nobleza sevillana. ${ }^{5} \mathrm{La}$ excepcional producción literaria de tema fernandino se explica por la magna campaña de canonización que durante un siglo aproximadamente vivió España y en cuya literatura se establecía una "Sevilla restaurada", ${ }^{6}$ marcando los cronistas una era feliz para la metrópoli de Andalucía que se iniciaba con su conquista por el rey San Fernando en 1248 y terminaba en 1671, año de su canonización por el papa Clemente X. ${ }^{7}$

La decadencia del imperio español en el siglo XVII y la pérdida de la hegemonía europea se hallaba necesitada de heroicas virtudes en sus monarcas que los panegiristas encontraron admirablemente en el Santo Rey, "caballero de Cristo", "ley viva de príncipes perfectos". Así, por ejemplo, fray Bartolomé García de Escañuela, en el prólogo a su Trono de glorias, se dirige a Carlos II y María Ana de Austria en estos términos: "En el mar de glorias de el Rey Don Fernando el Santo, Predecessor, y Abuelo de vuestras dos Reales Magestades de descubre la tierra de mi humilde estudio: en ella un Trono: en el Trono un Santo: todo igualmente pertenece indiuiso a vuestras dos Reales Magestades". ${ }^{8}$ No

5 P. Piñero, "La conquista de Sevilla en la poesía épica de los siglos de Oro", Archivo Hispalense, no. 171-173, 1973, pp. 162-163; F. Rodriguez Zapata, Glorias históricas y religiosas de San Fernando, su novena y biografía, sus principales cultos, con algunos elogios poéticos de tan augusto conquistador, Sevilla, 1874, hace el catálogo de los principales libros dedicados al santo (pp. 346 y ss.). Entre los más importantes fueron el de Juan de Pineda, Memorial de la excelente santidad y heroicas virtudes del Sr. R. D. Fernando III, Sevilla, 1627, así como el Fernando de la Torte Farfán, Fiesta de la Sta. Iglesia de Sevilla al culto nuevamente concedido al Sr. R. S. Fernando, Sevilla, 1671. Para la iconografía del santo vid L. Reau, Iconographie de l'art chrétien, III, Paris, 1955, p. 493, y J. Urrea, "San Fernando en Castilla y León", Boletín del Seminario de Estudios de Arte y Arqueología, Universidad de Valladolid, LXX, 1986, pp. 484-487.

6 J. A. de Vera y Figueroa, El Fernando o Sevilla restaurada, poema heroico escrito con los versos de la Gerusalemme Liberata del insigne Torquato Tasso ofrecido alla Magestad de Filippo IV el Grande monarca de España Emperador de las Indias, Milán, 1632.

7 D. Ortiz de Zúñiga, Annales eclesiásticos y seculares de la muy noble, y muy leal ciudad de Sevilla, metrópoli de la Andaluzía, que contienen sus más principales memorias desde el año 1246 en que emprendió conquistarla del poder de los Moros, el gloriosissimo Rey S. Fernando Tercero de Castilla, y León, hasta el de 1671, en que la Católica Iglesia le concedió el culto, y titulo de Bienaventurado, Madrid, 1677.

8 B. García de la Escañuela, op. cit. 
obstante, Fernando III de Castilla y de León, modelo de acrisoladas virtudes, no sólo era el glorioso antecesor del último de los Austrias sino también de algunos Grandes de España como don Gaspar Téllez Girón, duque de Osuna, según constaba genealógicamente por ocho líneas reales, y otras cuatro veces descendiente por la Casa de la Cerda, cuyas líneas eran fáciles de sacar en la Casa de los Duques de Medinaceli, como demostraba el cronista real Alonso Núñez de Castro. ${ }^{9}$

Este aspecto heroico y santo de la monarquía católica e hispana era el que interesaba resaltar en el gran retablo en piedra de la iglesia de San Fernando en México. También allí debían destacar plásticamente dos de las virtudes que habían sido cantadas por los literatos: la fe y la fortaleza. Fernando III el Santo había sido el introductor de los franciscanos en Castilla, su valedor en las nuevas tierras conquistadas a los musulmanes; él mismo era terciario franciscano y moriría con el hábito de San Francisco. ${ }^{10}$ El nuevo templo de la capital del Virreinato de la Nueva España se levantaba en el colegio donde se formaban los misioneros que habían de evangelizar California, Sierra Gorda y el Norte de México, donde las belicosas tribus chichimecas se resistían aún a cualquier contacto con los europeos. San Fernando, valedor de los franciscanos en España lo sería ahora espiritualmente en el Nuevo Mundo.

Aquel atuendo a la romana, que detestaba Interián de Ayala, resultaba el idóneo para la imagen de "miles christianus", pues era la visión de una monarquía católica, fuerte y poderosa, brazo derecho de la Fe y enérgico sostén de la Iglesia Católica, lo que predicaba aquella fachada retablo. La monarquía española, defensora de la fe contra turcos y herejes, evangelizadora de todo un continente, seguiría siendo también descendiente de San Fernando bajo la dinastía de los Borbones, como manifestaba el jesuita Antonio de Solis en su obra dedicada a Don Fernando Principe de Asturias. ${ }^{11}$

La imagen de los infieles entre llamas convenía a una sociedad en la que la amenaza de herejía era frecuente y en la que se dieron casos de falsas conversiones. Por otra parte, el mismo rey San Fernando solía

9 A. Núñez de Castro, op. cit.

10 M. de Manuel, Memorias para la vida del Santo Rey San Fernando, Madrid, 1800, y J. Gil de Zamora, Biografía del siglo XIV publicada por F. Fita, Boletín de la Real Academia de la Historia, Madrid, 1884.

11 A. de Solís, op. cit.: "Y yo de esta diria, que leera en ella V. Alteza, si ella logra tanto, no solo repetidas veces a su glorioso Avuelo San Fernando, y a su Augusto Padre el Señor Filipo Quinto, sino a todos sus Reales Ascendientes de la Real Casa de Castilla hasta el Rey Santo". 
conducir la leña para el suplicio de los herejes, según afirma el padre Flores. ${ }^{12}$ Aún no se habían apagado las hogueras de la Inquisición y el poder didáctico de la imagen competía con la fuerza aleccionadora de las palabras. Si en el pasado Santiago había sido el paladín de los conquistadores que veían mezquitas en los teocalli, en el presente San Fernando sería el paladín de los misioneros en la última fase de la conquista espiritual de México.

12 P. Florez, Reynas Catolic, t. I, f. 453, Apud J. Matute, Aparato para escribir la Historia de Triana, y de su iglesia parroquial, Sevilla, 1818, p. 133. 